\title{
Internal mammary artery conduit re-use in coronary artery bypass grafting: Case series and review
}

\author{
Carlos O. Encarnacion, Bartlomiej R. Imielski, Brandon D. Tomlin, Zahir A. Rashid \\ Division of Cardiothoracic Surgery, Medical College of Wisconsin, Milwaukee, WI, United States
}

Received: June 30, 2016

DOI: $10.5430 /$ css.v2n4p22
Accepted: July 24, $2016 \quad$ Online Published: July 27, 2016

URL: http://dx.doi.org/10.5430/css.v2n4p22

\begin{abstract}
Left internal mammary artery (LIMA) conduit is the gold standard graft for coronary artery bypass. Due to excellent patency rates with this graft it can safely be used in re-operative coronary bypass surgery. The safety of this technique is demonstrated with our report of re-used LIMA conduits in patients with extensive coronary artery disease (CAD). The success of this technique was evaluated by postoperative computerized tomographic angiography which demonstrated patent conduits and flow to the left anterior descending artery (LAD).
\end{abstract}

Key Words: Redo-coronary artery bypass grafting, Recycled left internal mammary artery, Re-used conduit, Coronary artery bypass grafting, Coronary revascularization

\section{INTRODUCTION}

The left internal mammary artery (LIMA) is the preferred conduit for left anterior descending artery (LAD) bypass revascularization. ${ }^{[1]}$ The recycling of the LIMA graft is an excellent option when there is concern for conduit availability. We present two consecutive patients who underwent redo-revascularization with re-use LIMA grafting.

\section{Case series}

Our first patient is a 66-year-old male with history of coronary artery disease (CAD) status post once vessel CABG in 1990, stent placement (left main and obtuse marginal artery) in 2000, end stage renal disease (ESRD) secondary to focal segmental glomerulosclerosis, status post renal transplant in 2007 requiring steroid therapy, hypertension (HTN), hy- perlipidemia (HLD), deep venous thrombosis, an factor $\mathrm{V}$ Leiden deficiency. Due to his allograft failure (on home peritoneal dialysis) he began pre-operative work-up for renal re-transplantation and was found to have extensive CAD.

He was found to have a $100 \%$ occlusion of the ostial LAD with an $80 \%$ stenosis at the Mid-LAD adjacent to the bifurcation of the second diagonal (D2) immediately distal to the LIMA anastomosis (see Figure 1A). The D2 also had a 70\% stenosis. Additionally, the left circumflex (LCX) had an $80 \%$ stenosis and the first OM1 had $80 \%$ in-stent stenosis. The posterior descending had moderate diffuse atherosclerosis. Based on these findings and his history, the decision was made to undergo CABG $\mathrm{x} 4$ with possible reuse of LIMA graft.

*Correspondence: Carlos O. Encarnacion, M.D.; Email: cencarnacion@mcw.edu; Address: Division of Cardiothoracic Surgery, Medical College of Wisconsin, 9200 W. Wisconsin Ave, Milwaukee, WI 53226, United States. 

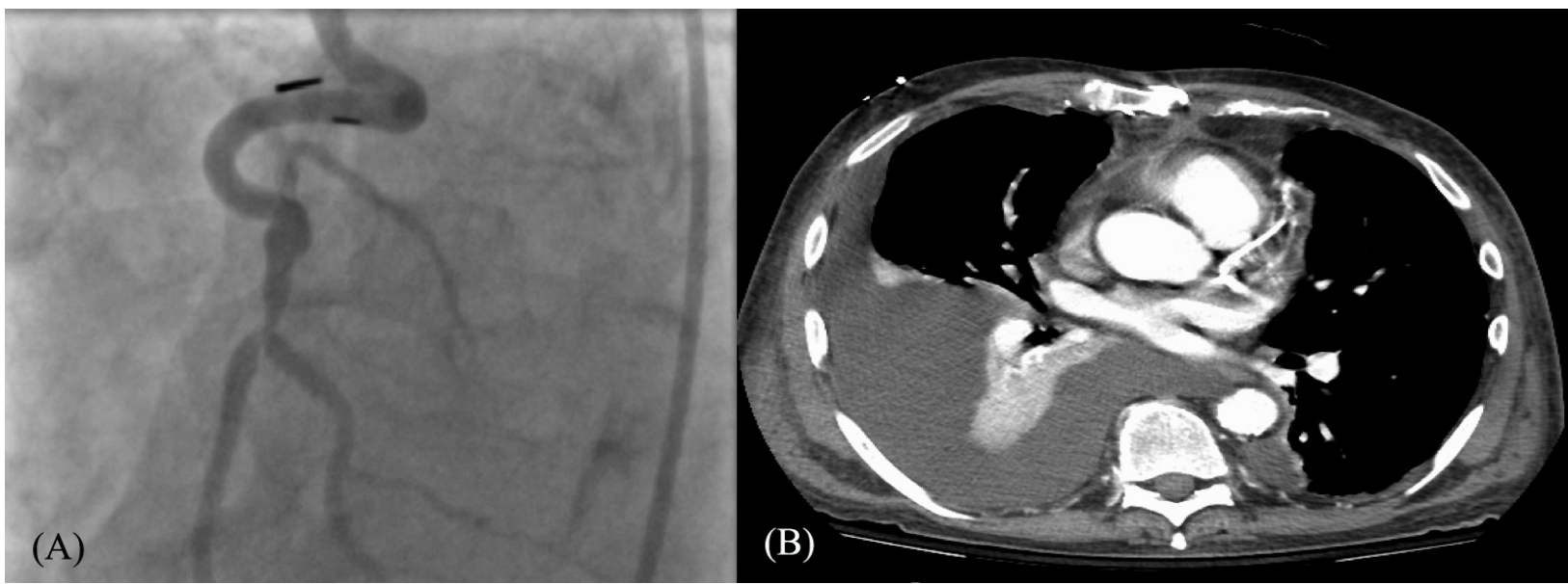

Figure 1. Patient 1

(A) Left image shows pre operative coronary angiography of LAD stenosis at LIMA anastomosis; (B) Right image displays postoperative computerized tomographic angiography of patent $L A D$
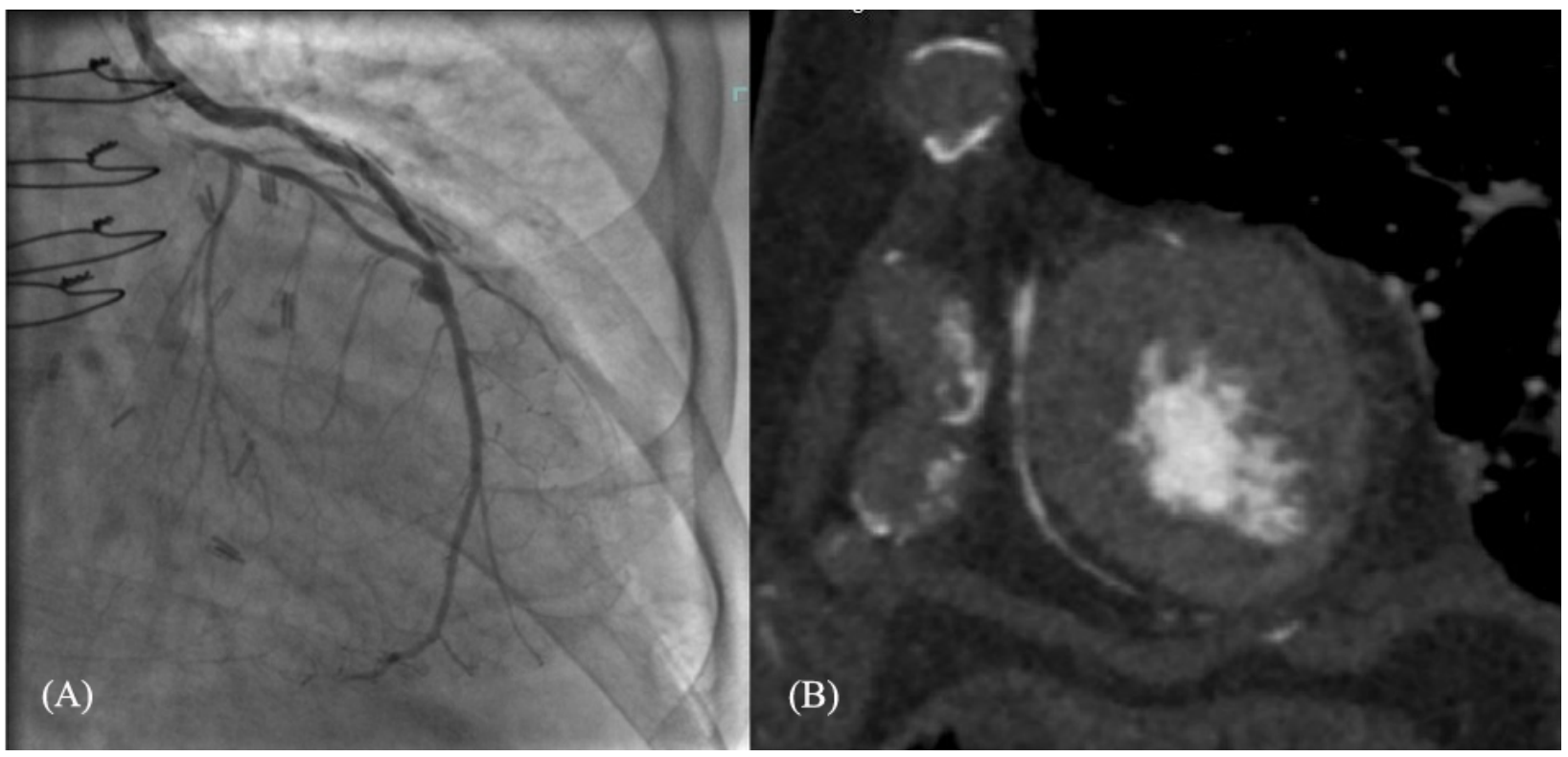

Figure 2. Patient 2

(A) Left side shows pre operative coronary angiography of LAD stenosis at LIMA anastomosis; (B) Right image displays postoperative computerized tomographic angiography of patent $L A D$

The second patient is a 72-year-old male with history of CAD status post 4 vessel CABG in 2006 following myocardial infarction, type two diabetes mellitus (DMII), ESRD on peritoneal dialysis, HTN, and HLD who presented on transfer from a referring hospital with chest tightness and nausea. On further workup he was found to have $100 \%$ occlusion in the mid-LAD (see Figure 2A), mid-LCX, mid-right coronary artery (RCA), with distal collateralization to the RCA and OM3.

Both procedures involved a redo sternotomy with dissection of the LIMA graft. After freeing an adequate length to extend the LIMA pedicle under the left sternal table, the right internal mammary artery (RIMA) was also harvested, in addition to the great saphenous vein $(\mathrm{SVG})$ from the left leg in the first patient and the right in the second. The patients were heparinized and placed on cardiopulmonary bypass without cardiac arrest via aortic and right atrial cannulation. The anastomoses of the LIMA to LAD were subsequently dissected out. The LIMAs were transected proximal to their coronary anastomosis. The stumps remaining were then over- 
sewn using a 7-0 Prolene running suture. In both cases the distal ends of the LIMAs were inspected for intimal disease and for adequate flow; once satisfied with their quality, each were respectively anastomosed to the new distal LAD targets with running 7-0 Prolene in an end-to-side fashion. In the first patient, the in-situ RIMA was anastomosed sequentially in end-to-side fashion to the OM1 artery and side-to-side fashion to D2. Finally, the reverse SVG was anastomosed from the ascending aorta to the right posterior descending artery (PDA). For the second patient, the RIMA was harvested as a free graft and anastomosed from the aorta to the D1 artery in an end-to-side fashion using 8-0 Prolene. The reverse SVG was anastomosed from the ascending aorta to the PDA as with the first patient. Both patients were taken off bypass without complication and closed in layers.

The total hospital length of stay was 5 and 15 days respectively. The first patient had an episode of atrial fibrillation during recovery but was chemically converted into sinus rhythm with amiodarone. The second patient's post-operative course was complicated by right pleural effusion requiring thoracentesis. Both patients followed up in the cardiothoracic surgery clinic for their fist postoperative visit 2 weeks after discharge without surgical complications and were cleared for cardiac rehabilitation.

\section{Discussion}

The LIMA to LAD graft is ideal for bypass surgery. ${ }^{[1,2]}$ The LIMA is a unique artery in that it is more resistant to atherosclerosis due to its resilient endothelium and nonfenestrated internal elastic lamina that is resistant to smooth muscle migration. ${ }^{[2]}$ In addition, the lumen of the LIMA is very close in size to that of the LAD, allowing for less turbulent flow after anastomosis. All of these reasons mean that the LIMA to LAD has a superior 10 year patency to any other coronary bypass graft. ${ }^{[1,2]}$ In situations where there is stenosis in the LAD distally to the original anastomosis, it would be ideal to take advantage of the well-documented benefits of this graft. Unfortunately, in re-operative CABGs adhesions around the LIMA can make it difficult to free and mobilize adequate length. Furthermore, during lysis of adhesion there is always risk of injury to the LIMA. The first patient was on chronic steroid and immunosuppressant's therapy prior to this surgery that may have helped reduce the amount of adhesions encountered. Nevertheless, a safe dissection was performed on the second patient in whom no chronic anti-inflammatory therapy was used.

Both patients did well in the short term. Though we do not yet have long-term outcomes for these patients, we have short term follow up of patent coronary flow evident in our follow up computerized tomographic angiography (see Figure $1 \mathrm{~B}$ and $2 \mathrm{~B}$ ) at 3 months and 2 months respectively. Angiographic evidence of patency was studied by Antona where the coronaries where studied between 7 and 35 months post operatively and all re-used vessels were found to be patent. ${ }^{[3]}$ In a contemporary study by Oumeiri recycling of LIMA graft had a $91 \%$ at 1 year and $83 \%$ at 5 years freedom from major cardiac events. ${ }^{[4]}$ Length of hospital stay and length of stay in intensive care unit were shown to be comparable to nonrecycled graft CABGs with 69 hour mean intensive care stay and 14 day mean hospital stay. ${ }^{[5]}$

The unusual medical history of these patients may have contributed to the success of our re-use of the LIMA graft; there may be more patients with similar histories in which this procedure can be considered. If conduit anastomosis need to be maximized and the LIMA graft does not reach the intended target a recycled free LIMA graft could be considered and has been reported as viable option in literature. ${ }^{[6]}$

\section{Conflicts of Interest Disclosure}

None.

\section{REFERENCES}

[1] Goldman S, Zadina K, Moritz T, et al. Long-term Patency of Saphenous Vein and Left Internal Mammary Artery Grafts after Coronary Artery Bypass Surgery. J Am Coll Cardiol. 2004; 44: 2149-2156. PMid: 15582312. http://dx.doi.org/10.1016/j.jacc. 2004 .08 .064

[2] Cheng A, Slaughter MS. How I choose conduits and configure grafts for my patients-rationales and practices. Ann of Cardiothorac Surg 2013; 2: 527-532. PMid: 23977632.

[3] Antona C, Parolari A, Zanobini M, et al. Midterm Angiographic Study of Five Recycled Mammary Arteries during Four Coronary Redos. Ann Thorac Surg. 1996; 61: 702-705. http://dx.doi .org $/ 10.1016 / 0003-4975(96) 89375-1$
[4] Oumeiri BE, Glineur D, Price J, et al. Recycling of Internal Thoracic Arteries in Reoperative Coronary Surgery: In-Hospital and Midterm Results. Ann Thorac Surg. 2011; 91: 1165-1168. PMid: 21440139. http://dx.doi.org/10.1016/j.athoracsur.2010.11.073

[5] Noirhomme PH, Underwood MJ, El Khoury GA, et al. Recycling of Arterial Grafts during Reoperative Coronary Artery Operations. Ann Thorac Surg. 1999; 67: 641-644. http://dx.doi.org/10.1016 /S0003-4975 (98) 01252-1

[6] Vivirito M, Conocchia M, Patanè R, et al. Free Internal Mammary Artery Graft Reimplantation on the Same Vessel in Repeat Coronary Revascularization. Tex Heart Inst J. 2015; 42: 162-165. PMid: 25873831. http://dx.doi.org/10.14503/THIJ-13-3845 